\title{
Antimicrobial agents and Burkholderia pseudomallei: perspectives from Thailand
}

\author{
Pawana Panomket \\ College of Medicine and Public Health, Ubon Ratchathani University, Ubon Ratchathani 34190, \\ Thailand
}

\begin{abstract}
Background: Burkholderia pseudomallei are the causative agent of melioidosis, a potentially life-threatening disease in humans and animals. It is a common public health threat in parts of Thailand.

Objective: To summarizes the current knowledge regarding antimicrobial agents and B. pseudomallei.

Methods: A literature search using MEDLINE (PubMed), SCOPUS, and OVID/LWWW databases.

Results: B. pseudomallei are intrinsically resistant to a wide range of antimicrobial agents including $\beta$-lactam antibiotics, aminoglycosides, and macrolides. Antimicrobial therapy for melioidosis is divided into an acute phase and an eradication phase. The current recommendations for the acute phase are parenteral antimicrobial agents for $\geq 10$ days using ceftazidime or a carbapenem. The eradication phase involves oral antimicrobial agents for $\geq 180$ days using trimethoprim-sulfamethoxazole. Amoxicillin-clavulanic acid may be used as an alternative. Ceftazidime revealed rare primary resistance and a high relapse rate.

Conclusion: Patients with acute melioidosis usually need intensive care and appropriate antibiotics for the acute and eradication phases. Ceftazidime is remains an effective agent in Thailand. A trend for decreasing susceptibility to antibiotics requires monitoring.
\end{abstract}

Keywords: Antibiotic resistance, Burkholderia pseudomallei, mechanisms of resistance, melioidosis

Burkholderia pseudomallei, an environmental Gram-negative bipolar bacillus, is the causative agent of melioidosis, a disease that is difficult to treat because of its diverse clinical presentations, frequent association with underlying disease, and recurrent nature [1]. Melioidosis is a major cause of communityacquired infections in endemic areas such as southeast Asia and northern Australia [2]. Though long present in the environment and also known to veterinarians, it is now increasingly recognized in other parts of the world [3]. Because relapses of melioidosis are common, clinical management and long courses of antibiotics are required for cure. B. pseudomallei have also been considered as biological warfare and/ or as bioterrorism agents, and are included in the category B list (US-CDC).

When B. pseudomallei infects a host, the host immune response is activated by innate cells and the bacteria are recognized by pattern recognition

Correspondence to: Pawana Panomket, College of Medicine and Public Health, Ubon Ratchathani University, Ubon Ratchathani 34190, Thailand.E-mail: panomketp@yahoo.com,mdpawapa@ ubu.ac.th receptors (PRR) located on these cells. Toll-like receptors (TLR) are a large family of PRR and normally, the PRR on innate cells are recognized by pathogen associated molecular patterns (PAMP) expressed on pathogens [4]. Like most Gramnegative bacteria, B. pseudomallei have a lot of PAMP, such as lipopolysaccharide (LPS), lipid-A, peptidoglycan, flagellin, type III secretion system (TTSS), and DNA [5]. Each PAMP is activated in a different kind of TLR for signaling transduction pathways. For example, LPS activates signals via TLR4 while flagellin activates signals via TLR5. However, LPS from B. pseudomallei activates signals via TLR2 and is different from that of other Gram-negative bacilli. An increased expression of TLR2 exists in patients with septic melioidosis [6]. Signal transduction pathways are divided into the Myd88-dependent pathway and the Myd88independent pathway. The products of the Myd88dependent pathway are inflammatory cytokines and the Myd88-independent pathway produces interferon (IFN)- $\beta$ and IFN-inducible gene products. Most IFN-inducible genes produce protective cytokines such as type I interferon. The functions of 
IFN- $\beta$ and IFN-inducible genes are antiviral and antiinflammatory. The activation of B. pseudomallei via TLR2 in the Myd88-dependent pathway indicates a protective role. However, the mechanism by which TLR2 signaling contributes to B. pseudomallei pathogenesis remains unclear. The Myd88independent pathway (or TANK-binding kinase-1; TBK1 dependent pathway) was studied using TBK1 deficient cells. The data showed the same bacterial counts in TBK1 deficient cells and control cells. Thus the Myd88-independent pathway is not able to control B. pseudomallei infection, but is able to control Escherichia coli and Brucella melitensis [7]. Type II interferon (IFN- $\gamma$ ) is produced within the first 24 hours of interaction between the host and $B$. pseudomallei and has an important role to play against B. pseudomallei. Other research found that mice can produce IFN- $\gamma$ resistant to infection [8]. The main functions of IFN- $\gamma$ are the activation of macrophages into highly powerful cytotoxic effector macrophages and the release of reactive oxygen intermediates (ROI) and reactive nitrogen intermediates (RNI) to destroy B. pseudomallei, and then the presentation of peptide antigen to T lymphocytes [9]. Neutrophils arrive at the site of the infection and secrete inflammatory cytokines and chemokines [10-12]. T cells and antibody play a role in the adaptive immune response. $\mathrm{CD}^{+}$and $\mathrm{CD}^{+} \mathrm{T}$ cells secrete IFN- $\gamma$ via $\mathrm{T}$ cells immunogens such as LolC, OppA, and PotF [13]. High IFN- $\gamma$ production contributes to a mouse's resistance to B. pseudomallei and the control of infection and cytotoxic $\mathrm{CD}^{+} \mathrm{T}$ cells defense bacteria by Granzyme B [13]. Antibodies are opsonins and these promote phagocytosis. High levels of B. pseudomallei-specific antibodies are found in patients with melioidosis during infection, such as IgG, IgM, and IgA antibodies [14]. The IgG isotype is a subclass of IgG1, which indicates Th1 responses [14]. However, a high incidence of relapse is also present. Therefore, the generation of these antibodies is ineffective in the prevention of melioidosis and at present there is no vaccine.

Our only present defense against $B$. pseudomallei are antimicrobials. However, B. pseudomallei are intrinsically resistant to many antibiotics, such as first and second-generation cephalosporins, macrolides, rifamycins, colistin, and aminoglycosides $[3,15]$. Antimicrobial agents effective against $B$. pseudomallei include amoxicillin/clavulanic acid (AMC), chloramphenicol, doxycycline, trimethoprimsulfamethoxazole (SXT), ureidopenicillins, ceftazidime, and carbapenems. Ceftazidime and carbapenem currently remain the main agents used for the acute intensive phase of therapy $[16,17]$.

\section{Antimicrobial agents and B. pseudomallei}

As melioidosis has a high mortality and relapse rates, antibiotic susceptibility to $B$. pseudomallei needs to be tested constantly wherever available. In a study at the Royal Darwin Hospital, $96 \%$ of 170 isolates of B. pseudomallei were found to be susceptible to meropenem/imipenem, ceftazidime, SXT, and doxycycline by agar dilution and $4 \%$ showed primary resistance. These included low-level resistance to SXT, ceftriaxone, amoxicillin/clavulanate, and doxycycline [18]. Thibault et al. found a single clinical isolate of $B$. pseudomallei that was resistant to ceftazidime, amoxicillin/clavulanate, and doxycycline [19]. However, this isolate was susceptible to imipenem. Antimicrobial resistance in clinical $B$. pseudomallei was surveyed in northeast Thailand through observed efficacy of antimicrobial drugs used to treat melioidosis. The results showed that 24 out of $4,021(0.6 \%)$ patients have one or more isolates resistant to ceftazidime $(n=8)$, amoxicillin/clavulanate $(n=4)$, or both drugs $(n=12)$. This finding indicated that B. pseudomallei associated with human infection, show rare primary resistance to ceftazidime or amoxicillin/clavulanate [20].

The current recommendations for antibiotic treatment are high-dose intravenous ceftazidime or a carbapenem administered for 10-14 days [16, 21]. Early treatment with ceftazidime can decrease mortality by about $50 \%$ [22]. Although ceftazidime is generally effective against B. pseudomallei, the recovery rate of patients is still low and high rates of relapses are found. The mechanism of relapse is still unclear. One factor which may be related to the reduction of antimicrobial activity is the presence of biofilms. B. pseudomallei are bacteria of special interest because they grow preferentially in microcolonies and biofilms both in vitro and in vivo in an animal model [23]. The morphologic data obtained in this study confirm that a B. pseudomallei bacterium can produce exopolysaccharide materials that constitute a highly hydrated glycocalyx [23]. The production of glycocalyces can facilitate the formation of microcolonies, allowing bacteria to adhere to the surface. The capacity of B. pseudomallei to produce a biofilm varies in quantity in each isolate and it was found that there is no correlation between biofilm 
production and the source of isolation, including the virulence of the bacteria [24]. When B. pseudomallei are grown in conditions that induce biofilm formation, they are resistant to all antimicrobial agents. By contrast, all planktonic isolates are susceptible to all agents. Ceftazidime and SXT were studied for resistance of B. pseudomallei compared with planktonic isolates and biofilm conditions. The minimal inhibitory concentrations (MIC) of ceftazidime and SXT for the planktonic cells of B. pseudomallei were 4 and $40 \mu \mathrm{g} / \mathrm{ml}$ respectively, while the minimum bactericidal concentrations (MBC) were 8 and 3,000 $\mathrm{g} / \mathrm{ml}$ respectively. When $B$. pseudomallei biofilms were treated with $0,25,50,100$, and 200 times of the MICs of ceftazidime and SXT in Mueller-Hinton broth in a sessile minimum biofilm eliminating concentration testing device, a large number of cells remained viable at 12 hours and an almost equally large number were still active at 24 hours, except in the biofilm treated with 200 times the MIC of ceftazidime in which viable cells decreased at 24 hours [25]. The very high minimum biofilm eliminating concentration explains the lack of success in the treatment of chronic manifestations of $B$. pseudomallei infection. In addition, the biofilm mutant strains are resistant to all antimicrobial agents when induced to form biofilm [26].

The formation of biofilm is a multistep process that requires the participation of structural appendages such as flagella, type IV pili, and quorum sensing. Quorum-sensing is a population density-mediated form of cell-cell communication via the production of the signaling molecule. Cyclic diguanylic acid (c-di-GMP) is an intracellular signaling molecule involved in the regulation of biofilm formation. The higher intracellular c-di-GMP levels in the cdpA null mutant are associated with the increased production of exopolysaccharides and increased cell to cell aggregation, and biofilm formation [27]. Bacterial biofilm is believed to be an optimum site for the activation of quorum-sensing because it is here that natural populations are at their highest cell densities. Sigma factor $\sigma^{\mathrm{E}}(\mathrm{RpoE})$ is a prokaryotic transcription initiation factor that enables specific binding of RNA polymerase to gene promoters. Inactivation of the rpoE operon changes the B. pseudomallei phenotype, including increased susceptibility to killing by menadione and $\mathrm{H}_{2} \mathrm{O}_{2}$, susceptibility to high osmolarity, reduced ability to form biofilms, and reduced survival in macrophages. Therefore, this explains the expression of $r p o E$ related to $B$. pseudomallei adaptation to adverse environmental conditions, especially environmental stress tolerance and biofilm formation [28]. B. pseudomallei in biofilm cells were reported as highly resistant to ceftazidime, doxycycline, imipenem, and SXT. The possible drug-resistant mechanism of biofilm is still unclear. However, research found that stimulation of the bacteria to produce biofilm results in upregulation of some genes to be resistant against antimicrobial agents [26]. The protein expression study in B. pseudomallei wild-type and biofilm defective mutants in biofilm stimulating conditions, revealed a different protein expression under biofilm stimulating conditions. This indicates that up- or downregulated protein may be involved in biofilm formation and may also play a key role in antimicrobial resistance [29].

$\beta$-Lactam resistance of Gram-negative bacilli is a problem. The most important mechanism is the production of chromosomally-mediated $\beta$-lactamases. However, mutants in $\beta$-lactamases are naturally produced at high levels. At present, ceftazidime is the antibiotic of choice for the treatment of melioidosis, but ceftazidime-resistant B. pseudomallei are found. To illuminate this, a class D $\beta$-lactamases gene from B. pseudomallei was cloned and sequenced. The encoded enzyme was an oxacillinase. The high minimum inhibitory concentration (MIC) observed in B. pseudomallei is the result of a class $\mathrm{D} \beta$-lactamase gene [30]. This consideration may assist in finding solutions to ceftazidime-resistant B. pseudomallei in the future. The complete genome sequence of $B$. pseudomallei revealed seven genes encoding Ambler class $\mathrm{A}, \mathrm{B}$, and $\mathrm{D} \beta$-lactamases. The gene encoded by blaA (or penA; Ambler class A) hydrolyzed most cephalosporins, but were inhibited by clavulanate $[3,31,32]$. Acquired resistance to $\beta$-lactam antibiotics may have occurred during treatment with the combination of a $\beta$-lactam- $\beta$-lactamase inhibitor. The study of loss of penicillin-binding protein 3 (PBP3) involved a comparison of susceptible ceftazidime and resistant ceftazidime by using molecular techniques. Mutagenesis analyzed large scale genomic loss, involving 49 genes in B. pseudomallei-resistant strains. The results represented the deletion of a gene encoding penicillin binding protein 3 within the region of genomic loss [33]. The loss of PBP3 allows the appearance of a filamentous state by gram stain and poor growth. Inactivation of PBP3 results in the inhibition of cell division and growth into long filaments. 
Other agents effective against B. pseudomallei include a number of novel antiinfective agents that are effective against antibiotic-resistant $B$. pseudomallei. Antimicrobial peptides (AMP) possess potent broad-spectrum bactericidal activities [34]. Ten AMP, including histatin 5 and histatin variants, human cathelicidin peptide LL-37 and lactoferrin peptides, were tested for in vitro activities against B. pseudomallei. LL-37 exhibited the most effective activity against B. pseudomallei [35]. Moreover, natural products from plants were tested to screen for antimicrobial activity to B. pseudomallei. A methanolic extract from Barringtonia acutangula (L.) Gaertn. showed the best antimicrobial results against B. pseudomallei with an inhibition zone of 18 $\mathrm{mm}$, and MIC and minimal bactericidal concentration (MBC) of $4 \mathrm{mg} / \mathrm{ml}$ [36].

Other treatment modalities for melioidosis include combination immunotherapy using granulocyte colony stimulating factor (G-CSF) with antibiotics, which reduced the mortality rate of patients with septic shock in Australia [37]. However, the report was not a prospective randomized-controlled trial. It was conducted at the Royal Darwin Hospital, the site of an excellent intensive care unit, which may have been at least partly responsible for the better survival rate. A murine model for melioidosis that allows adjunct G-CSF with ceftazidime and ceftazidime alone treatment was investigated and found to be not significantly different [38]. The authors concluded that the use of G-CSF is of limited benefit. However, G-CSF has been shown to improve the outcomes of sepsis in an animal model and to improve neutrophil function in vitro [37]. Therefore, combination with G-CSF may benefit in cases of neutrophil function defects. A randomized controlled trial of G-CSF was conducted in Thailand for severe sepsis resulting from melioidosis. The researchers compared ceftazidime plus G-CSF with ceftazidime alone. The study showed similar mortality rates in both groups [39]. Panomket et al. found that the use of low dose steroids as an adjunct in the treatment of murine melioidosis does not provide benefits and may have negative effects in treatment of humans with diabetic mellitus [40]. In this study, diabetic mice infected with B. pseudomallei were given hydrocortisone-ceftazidime and showed an increase in their blood glucose and a reduced rate of survival.

\section{Conclusion}

Ceftazidime remains the drug of choice for treating melioidosis, but B. pseudomallei are developing resistance to this agent. Although resistance to ceftazidime is still rare, a longer course of treatment and treatment for recurrences may be required.

\section{Acknowledgments}

The authors thank Bob Tremayne, Division of International Relations, Ubon Ratchathani University for editing the English of the manuscript. The authors have no conflict of interest to declare.

\section{References}

1. Chaowagul W, White NJ, Dance DA, Wattanagoon Y, Naigowit P, Davis TM, et al. Melioidosis: a major cause of community-acquired septicemia in northeastern Thailand. J Infect Dis. 1989; 159:890-99.

2. Leelarasamee A, Bovornkitti S. Melioidosis: review and update. Rev Infect Dis. 1989; 11:413-25.

3. Cheng AC, Currie BJ. Melioidosis: epidemiology, pathophysiology, and management. Clin Microbiol Rev. 2005; 18:383-416.

4. Akira S, Uematsu S, Takeuchi O. Pathogen recognition and innate immunity. Cell. 2006; 124:783-801.

5. Wiersinga WJ, van der Poll $\mathrm{T}$. Immunity to Burkholderia pseudomallei. Curr Opin Infect Dis. 2009; 22:102-8.

6. Wiersinga W, Wieland C, Dessing M, Chantratita N, Cheng AC. Toll-like receptor 2 impairs host defense in gram-negative sepsis caused by Burkholderia pseudomallei (Melioidosis). PLoS Med. 2007; 4:e248.

7. Panomket P, Splitter G, Harms J, Sermswan RW, Chedchotisakd P, Wongratanacheewin S. TBK1 does not play a role in the control of in vitro Burkholderia pseudomallei growth. Trans R Soc Trop Med Hyg. 2008; 102 (suppl 1):S95-100.

8. Santanirand P, Harley VS, Dance DA, Drasar BS, Bancroft GJ. Obligatory role of gamma interferon for host survival in a murine model of infection with Burkholderia pseudomallei. Infect Immun. 1999; 67: 3593-600.

9. Taylor PR, Martinez-Pomares L, Stacey M, Lin HH, Brown GD, Gordon S. Macrophage receptor and immune recognition. Ann Rev Immunol. 2005; 23: 901-44.

10. Martineau AR, Newton SM, Wilkinson KA, Kampmann B, Hall BM, Nawroly N, et al. Phenotypic and functional characterization of human memory $\mathrm{T}$ cell responses to Burkholderia pseudomallei. PloS Negl Trop Dis. 
2009; 3:e407.

11. Scapini P, Lapinet-Vera JA, Gasperini S, Calzetti F, Bazzoni F, Cassatella MA. The neutrophil as a cellular source of chemokines. Immunol Rev. 2000; 177: 195-203.

12. Segal AW. How neutrophils kill microbes. Ann Rev Immunol. 2004; 23:197-223.

13. Tippayawat P, Saenwongsa W, Mahawantung J, Suwannasaen D, Chetchotisakd P, Limmathurotsakul $\mathrm{D}$, et al. Phenotypic and functional characterization of human memory T cell responses to Burkholderia pseudomallei. PLoS Negl Trop Dis. 2009; 3:e407.

14. Vasu C, Vadivelu J, Puthucheary SD. The humoral immune response in melioidosis patients during therapy. Infection. 2003; 31:24-30.

15. White NJ. Melioidosis. Lancet. 2003; 361:1715-22.

16. Chaowagul W. Recent advances in the treatment of severe melioidosis. Acta Tropica. 2000; 74:133-37.

17. Chierakul W, Anunnatsiri S, Short JM, Maharjan B, Mootsikapun P, Simpson Andrew JH, et al. Two randomized controlled trials of ceftazidime alone versus ceftazidime in combination with trimethoprimsulfamethoxazole for the treatment of severe melioidosis. Clin Infect Dis. 2005; 41:1105-13.

18. Jenny AWJ, Lum G, Fisher DA, Currie BJ. Antibiotic susceptibility of Burkholderia pseudomallei from tropical northern Australia and implications for therapy of melioidosis. Int J Antimicrob Agents. 2001; 17: 109-13.

19. Thibault FM, Hernandez E, Vidal DR, Girardet M, Cavallo JD. Antibiotics susceptibility of 65 isolates of Burkholderia pseudomallei and Burkholderia mallei to 35 antimicrobial agents. JAC. 2004; 54: 1134-8.

20. Wuthiekanun V, Amornchai P, Saiprom N, Chantratita N, Chierakul W, Koh GCKW, et al. Survey of antimicrobial resistance in clinical Burkholderia pseudomallei isolates over two decades in northeast Thailand. Antimicrob Agents Chemother. 2011; 11: 5388-91.

21. Dance DA. Melioidosis. Curr Opin Infect Dis. 2002; 15:127-32.

22. White NJ, Dance DA, Chaowagul W, Wattanagoon Y, Wuthiekanun V, Pitakwatchara N. Halving of mortality of severe melioidosis by ceftazidime. Lancet. 1989;2: $697-701$.

23. Vorachit M, Lam K, Jayanetra P, Costerton JW. Electron microscopy study of the mode of growth of Pseudomonas pseudomallei in vitro and in vivo. $\mathrm{J}$ Trop Med Hyg. 1995; 98:379-91.
24. Taweechaisupapong S, Kaewpa C, Arunyanart C, Kanla P, Homchumpa P. Virulence of Burkholderia pseudomallei does not correlate with biofilm formation. Microb Pathog. 2005; 39:77-85.

25. Vorachit M, Lam K, Jayanetra P, Costerton JW. Resistance of Pseudomonas pseudomallei growing as a biofilm on silastic disc to ceftazidime and cotrimoxazole. Antimicrob Agents Chemother. 1993; 37: 2000-2.

26. Sawasdidoln C, Taweechaisupapong S, Sermswan RW, Tattawasart U, Tungpradabkul S, Wongratanacheewin S. Growing Burkholderia pseudomallei in biofilm stimulating conditions significantly induces antimicrobial resistance. PLoS One. 2010; 5:e9196.

27. Lee HS, Gu F, Ching SM, Lam Y, Chua KL. CdpA, a Burkholderia pseudomallei cyclic-di-GMP phosphodiesterase involved in autoaggregation, flagella synthesis, motility, biofilm formation, cell invasion and cytotoxicity. Infect immune. 2010; 78: $1832-40$.

28. Korbsrisate S, Vanaporn M, Kerdsuk P, Kespichayawattana W, Vattanaviboon P, Kiatpapan P, et al. The Burkholderia pseudomallei RpoE (AIgU) operon is involved in environmental stress tolerance and biofilm formation. FEMS Microbial Lett. 2005; 252:243-9.

29. Puknum A, Tungpradabkul S, Taweechaisupapong S. Proteomic study of Burkholderia pseudomallei wild type and biofilm defective mutant in biofilm stimulating condition. Poster presentation on Commission on Higher Education Congress III University Staff Development. 2010.

30. Niumsup P, Wuthiekanun V. Cloning of the class D lactamase gene from Burkholderia pseudomallei and studies on its expression in ceftazidime susceptible and resistant strains. JAC. 2002; 50:445-55.

31. Livermore DM, Chau PY, Wong, AI, Leung YK. $\beta$-Lactamase of Pseudomonas pseudomallei and its contribution to antibiotic resistance. J Antimicrob Chemother. 1987; 20:313-21.

32. Cheung TK, Ho PL, Woo PC, Yuen KY, Chau PY. Cloning and expression of class A $\beta$-lactamase gene bla $_{\mathrm{BPS}}$ in Burkholderia pseudomallei. Antimicrob Agents Chemother. 2002; 46:1132-5.

33. Chantratita N, Rholl DA, Sim B, Wuthiekanum V, Limmathurotsakul D, Amornchai P, et al. Antimicrobial resistance to ceftazidime involving loss of penicillinbinding protein 3 in Burkholderia pseudomallei. PNAS. 2012; 108:17165-70.

34. Davidson RN, Griffiths CJ, Wilkinson RJ. Neutrophil 
mediated innate immune resistance to mycobacteria. J Clin Invest. 2007; 117:1988-94.

35. Kanthawong S, Nazmi K, Wongratanacheewin S, Bolscher JGM, Wuthiekanun V, Taweechaisupapong S. In vitro susceptibility of Burkholderia pseudomallei to antimicrobial peptides. Int J Antimicrob Agents. 2009; 34:309-14.

36. Panomket P, Wanrum S, Srivoramas T, Prongprom N Bioactivity of plant extracts against Burkholderia pseudomallei. Asian Biomed. 2012; 6:619-23.

37. Cheng AC, Stephens DP, Anstey NM, Currie BJ. Adjunctive granulocyte colony-stimulating factor for treatment of septic shock due to melioidosis. Clin Infect Dis. 2004; 38:32-7.

38. Powell K, Ulett G, Hirst R, Norton R. G-CSF immuno- therapy for treatment of acute disseminated murine melioidosis. FEMS Microbiol Lett. 2003; 224:315-8.

39. Cheng AC, Limmathurotsakul D, Chierakul W, Getchalarat N, Wuthiekanun V, Stephens DP, et al. A randomized controlled trial of granulocyte colonystimulating factor for the treatment of severe sepsis due to melioidosis in Thailand. Clin Infect Dis. 2007; 45:308-14.

40. Panomket P, Chetchotisakd P, Sermswan RW, Pannengpetch P, Wongratanacheewin S. Use of low dose steroid as an adjunct in the treatment, in mice, of severe sepsis caused by Burkholderia pseudomallei. Ann Trop Med Parasitol. 2009; 103: 635-46. 